\title{
MANIFOLDS OF RIEMANNIAN METRICS WITH PRESCRIBED SCALAR CURVATURE
}

\author{
BY ARTHUR E. FISCHER AND JERROLD E. MARSDEN ${ }^{1}$
}

Communicated by S. S. Chern, August 1, 1973

1. Introduction. Throughout, $M$ will denote a $C^{\infty}$ compact connected oriented $n$-manifold, $n \geqq 2$. Let $\rho: M \rightarrow R$ be a $C^{\infty}$ function, $\mathscr{M}$ the space of $C^{\infty}$ riemannian metrics on $M$ and

$$
\mathscr{M}_{\rho}=\{g \in \mathscr{M}: R(g)=\rho\}
$$

where $R(g)$ is the scalar curvature of $g$. As in Ebin [3], a superscript $s$ will denote objects in the corresponding Sobolev space, $s>n / 2+1$ (one can also treat $W^{s, p}$ spaces in the same way), and we also allow $s=\infty$ so $\mathscr{M}^{\infty}=$ $\mathscr{M}$. Sign conventions on curvatures are as in Lichnerowicz [10].

Two of our main results follow:

THEOREM 1. If $\rho$ is not identically zero or a positive constant, then $\mathscr{M}_{s}^{\rho}$ is a smooth submanifold of $\mathscr{M}^{s}$.

We can also treat the case $\rho \equiv 0$. Let $\mathscr{F}^{s}$ denote the set of flat metrics in $\mathscr{M}^{s}$. Then we have

TheOREM 2. Assume $\mathscr{F}^{s} \neq \varnothing$. Writing $\mathscr{M}_{0}^{s}=\left(\mathscr{M}_{0}^{s} \mid \mathscr{F} s\right) \cup \mathscr{F}^{s}, \mathscr{M}_{0}^{s}$ is the disjoint union of closed submanifolds.

REMARK. If $\operatorname{dim} M=2, \mathscr{M}_{0}^{s}=\mathscr{F}^{s}$, and if $\operatorname{dim} M=3$, the hypothesis that $\mathscr{F}^{s} \neq \varnothing$ can be dropped.

The proof of Theorem 1 also allows us to conclude that a solution $h$ of the linearized equations $D R\left(g_{0}\right) \cdot h=0$ is tangent to a curve of exact solutions of $R(g)=\rho$ through a given solution $g_{0}$, provided $\rho$ is not a constant $\geqq 0$. In the terminology of [4] we say the equation $R(g)=\rho$ is linearization-stable at $g_{0}$. From Theorem 3 below the equation $R(g)=0$ is still linearization-stable about a solution $g_{0}$ provided $\operatorname{Ric}\left(g_{0}\right)$ is not identically zero.

For the singular case $\rho=0$, Theorem 2 incorporates an isolation theorem inspired by the work of Brill and Deser [2], namely, that the flat metrics are isolated solutions of $R(g)=0$. As a corollary one has: If $g(t)$ is a

AMS (MOS) subject classifications (1970). Primary 53C25, 58D15; Secondary 35J60, 58G99, 47H15.

Key words and phrases. Scalar curvature, submanifolds of riemannian metrics, flat metrics, isolated metrics, infinitesimal deformations, stability of linearized equations.

${ }^{1}$ The authors were partially supported by NSF grants GP-39060 and GP-15735. 
continuous curve in $\mathscr{M}, R(g(t))=0$ and if $g(0) \in \mathscr{F}$, then $g(t) \in \mathscr{F}$. In the course of proving Theorem 2 , we shall also prove that the space $\mathscr{F}$ has the structure of a homogeneous fiber bundle with finite-dimensional fiber (Theorem 4).

Details and extensions of the present work will appear in [5]. We thank J. Bourguignon, D. Ebin, J. Kazdan, R. Palais, F. Warner, A. Weinstein, and $\mathbf{J}$. A. Wolf for useful conversations.

2. Local surjectivity of the scalar curvature map. In the following we let $H^{s}$ denote the $H^{s}$ maps from $M$ to $R$, and $S_{2}^{s}$ the $H^{s}$ 2-covariant symmetric tensors. It is easy to see that $R: \mathscr{M}^{s} \rightarrow H^{s-2}$ is a $C^{\infty}$ map.

Theorem 1 is a special case of part (ii) of the next result in case $\rho$ is not a positive constant (see Remark 1 below).

THEOREM 3. Let $g \in \mathscr{M}^{s}$ and suppose that

(i) if $R(g)=0$ then $\operatorname{Ric}(g)$, the Ricci tensor, is not identically zero and

(ii) $R(g) /(n-1)$ is not a positive constant equaling an eigenvalue of $\Delta_{g}$, the Laplace-Beltrami operator on functions.

Then $R: \mathscr{M}^{s} \rightarrow H^{s-2}$ maps any neighborhood of $g$ onto a neighborhood of $R(g)$.

SKETCH OF PROOF. We shall assume $s<\infty$; the case $s=\infty$ requires a regularity argument as in Ebin [3]. It then suffices by the standard implicitfunction theorem to show that $\gamma_{g}=D R(g): S_{2}^{s} \rightarrow H^{s-2}$ is surjective.

A classical computation (see. e.g., [10]) gives

$$
\gamma_{g}(h)=\Delta(\operatorname{tr} h)+\delta \delta h-h \cdot \operatorname{Ric}(g),
$$

where $\operatorname{tr}$ is the trace, $\delta \delta$ is the double covariant divergence, and $\Delta=\Delta_{g}$. The $L_{2}$-adjoint of $\gamma_{g}$ is $\gamma_{g}^{*}: H^{s} \rightarrow S_{2}^{s-2}$,

$$
\gamma_{o}^{*}(f)=g \Delta f+\text { Hess } f-f \operatorname{Ric}(g),
$$

where Hess $f=\nabla \nabla f$ is the Hessian of $f$. Since $\gamma_{g}^{*}$ has injective symbol, from elliptic theory it suffices to show that $\gamma_{g}^{*}$ is injective (see, e.g., Berger and Ebin [1]). Assume $\gamma_{g}^{*} f=0$. The trace of this yields $(n-1) \Delta f=$ $R(g) f$. Thus $R(g)=0$ implies $f$ is constant and so $\gamma_{o}^{*} f=-f \operatorname{Ric}(g)=0$. Since $\operatorname{Ric}(g) \neq 0, f=0$. This takes care of case (i). Consider now (ii). Taking the divergence of $\gamma_{g}^{*} f=0$ and using the identities $\delta$ Hess $f-d \Delta f+d f \cdot \operatorname{Ric}(g)=$ 0 and $\delta(\operatorname{Ric}(g))=-\frac{1}{2} d(R(g))$, we get $\frac{1}{2} f d(R(g))=0$. Suppose $f \neq 0$. If $f$ is never zero, $d R(g)=0$ so $R(g)$ is a constant, which, together with $(n-1) \Delta f=$ $R(g) f$, contradicts (ii). Thus find $x_{0} \in M$ such that $f\left(x_{0}\right)=0$. We must have $d f\left(x_{0}\right) \neq 0$; indeed, if $d f\left(x_{0}\right)=0$, let $\gamma(t)$ be a geodesic starting at $x_{0}$ and $h(t)=f(\gamma(t))$. From $\gamma_{g}^{*} f=0$ and $(n-1) \Delta f=R(g) f$ we find Hess $f=$ $(\operatorname{Ric}(g)-1 /(n-1) g R(g)) f$ and hence $h(t)$ satisfies the linear second-order 
differential equation

$$
\begin{aligned}
h^{\prime \prime}(t) & =(\operatorname{Hess} f)_{\gamma(t)} \cdot\left(\gamma^{\prime}(t), \gamma^{\prime}(t)\right) \\
& =\left(\left(\operatorname{Ric}(g)-\frac{1}{n-1} g R(g)\right)_{\gamma(t)}\left(\gamma^{\prime}(t), \gamma^{\prime}(t)\right)\right) h(t)
\end{aligned}
$$

with $h(0)=0$ and $h^{\prime}(0)=0$. Thus $h(t)=0$ and hence $f=0$. Thus 0 is a regular value of $f$ and hence $d(R(g))=0$ on an open dense set and hence everywhere. Thus again $R(g)=$ constant, contradicting (ii). Thus $f=0$ and $\gamma_{g}^{*}$ is injective.

Note. We thank J. P. Bourguignon for pointing out that $\gamma_{g}^{*} f=0, f \neq 0$, implies $R(g)=$ constant. Previously we had (ii) replaced with the condition that $R(g) \leqq 0$.

REMARKS. 1. If $\operatorname{Ric}(g)=0$, the kernel of $\gamma_{g}^{*}$ is the set of constant functions so surjectivity of $\gamma_{g}$ fails. Similarly, on a standard sphere in $\boldsymbol{R}^{n+1}$ of radius $r_{0}$, the first order spherical harmonics (solutions of $\Delta f=n f / r_{0}^{2}$ ) also satisfy

$$
\text { Hess } f=(\operatorname{Ric}(g)-R(g) g /(n-1)) f=-g f / r_{0}^{2}
$$

and so again $\gamma_{g}$ is not surjective.

It is in fact reasonable to conjecture that these are the only cases in which $\gamma_{g}$ fails to be surjective. For example, among the spaces whose scalar curvature is a positive constant, it follows using a theorem of Obata's [13] that for those spaces with parallel Ricci tensors (e.g., products of Einstein spaces), $\gamma_{g}$ is surjective unless the space is a standard sphere.

2. J. Kazdan and F. Warner have pointed out that Theorem 3 for $W^{s, p}$ spaces can be used to yield some of their results concerning what functions can be realized as scalar curvatures ([8], [9]). This will be carried out in a note following [5]. A variant of this technique yields: If $n \geqq 3$ and there is a $g \in \mathscr{M}^{s}$ with $R(g)=0, \operatorname{Ric}(g) \neq 0$ then $R: \mathscr{M}^{s} \rightarrow H^{s-2}$ is surjective; i.e., every function can be realized as a scalar curvature of some metric. This follows from local surjectivity of $R$, together with [9, Theorem 4.3]. For $n=2$, the map $g \mapsto R(g)$ cannot be onto a neighborhood of $R\left(g_{0}\right)=0$, since then $M=T^{2}$ which by the Gauss-Bonnet theorem has no metric with Gaussian curvature strictly positive or strictly negative.

3. Note that we are not making any statements about whether or not $\mathscr{M}_{\rho}^{s}$ is empty. For example, Lichnerowicz [11] has shown that for certain spin manifolds, $\mathscr{M}_{\rho}^{s}=\varnothing$ if $\rho$ is positive. However, Kazdan and Warner ([8], [9]) have shown that if $n \geqq 3$ and $\rho$ is negative somewhere, or if $n=2$ and $\rho$ satisfies a sign condition consistent with the Gauss-Bonnet formula, then $\mathscr{M}_{\rho}^{s} \neq \varnothing$.

Set $\mathscr{E}_{0}^{s}=\left\{g \in \mathscr{M}^{s}: \operatorname{Ric}(g)=0\right\}$, the space of Ricci-flat metrics. Then Theorem 3 implies that $\mathscr{M}_{0}^{s} \mid \mathscr{E}_{0}^{s}$ is a $C^{\infty}$ submanifold of $\mathscr{M}^{s}$. From FischerWolf ([6], [7]) we have that if $\mathscr{F}^{s} \neq \varnothing$, then $\mathscr{E}_{0}^{s}=\mathscr{F} s$; i.e., every Ricci-flat 
metric is flat. Thus the essential assertion of Theorem 2 is that $\mathscr{M}_{0}^{s} \mid \mathscr{F}^{s}=$ $\mathscr{M}_{0}^{s} \mid \mathscr{E}_{0}^{s}$ is closed and that $\mathscr{F}^{s}$ is a manifold. This is discussed next.

3. Flat metrics and metrics with zero scalar curvature. We begin with the structure of $\mathscr{F} s$. Let $\mathscr{H}^{s-1}$ denote the $H^{s-1}$ flat riemannian connections on $M$. For $H \in \mathscr{H}^{s-1}$ let $I_{H}^{s+1} \subset \mathscr{D}^{s+1}$ (the $H^{s+1}$ diffeomorphisms of $M$ ) denote the Lie group of affine transformations of $H$. Let $\mathscr{F}_{H}^{s}$ denote the $H^{s}$ flat riemannian metrics whose Levi-Civita connection is $H$. Then $I_{H}^{s+1}$ acts by pullback on $\mathscr{F}_{H}^{s}, A: I_{H}^{s+1} \times \mathscr{F}_{H}^{s} \rightarrow \mathscr{F}_{H}^{s}$, and the action is continuous.

THEOREM 4. The space $\mathscr{H}^{\text {s-1 }}$ is homeomorphic to the homogeneous space $\mathscr{D}^{s+1} / I_{H}^{s+1}$. Using the above action $A$, the associated homogeneous fiber bundle is

$$
\pi: \mathscr{F}^{s} \rightarrow \mathscr{H}^{s-1} \approx \mathscr{D}^{s+1} / I_{H}^{s+1},
$$

where $\pi(g)$ is the Levi-Civita connection of $g$, and the fibers $\pi^{-1}(H)=\mathscr{F}_{H}^{s}$ are finite-dimensional manifolds. Thus $\mathscr{F} s$ is the total space of a homogeneous fiber bundle, and moreover, $\mathscr{F}^{s}$ is a smooth submanifold of $\mathscr{M}^{s}$.

SKeTCH OF PROOF. That $\mathscr{D}^{s+1}$ acts transitively on $\mathscr{H}^{s-1}$ follows as in Wolf [14, Theorem 3.3.1]. To see that $\mathscr{F}_{H}^{s}$ is finite-dimensional note that it is in one-to-one correspondence with the $\Psi_{x}$-invariant inner products on $T_{x} M$, where $x \in M$ and $\Psi_{x}$ is the linear holonomy group of $H$ at $x$ (Wolf [14, Theorem 3.4.5]). The last assertion follows from the fact that $\mathscr{F} s$ is the orbit of $\mathscr{F}_{H}^{\infty}$ under $\mathscr{D}^{s+1}$.

Our next theorem shows that near a flat metric, there exists no metric with scalar curvature $R(g) \geqq 0, R(g) \not \equiv 0$, and that the flat solutions of $R(g)=0$ are isolated solutions. This result extends to a full neighborhood of the flat metrics the "second order" version of these results in [2] and [9].

Theorem 5. Let $g_{F} \in \mathscr{F}:$ Then there exists a neighborhood $U \subset \mathscr{M}^{*}$ of $g_{F}$ such that if $g \in U$ and $R(g) \geqq 0$, then $g$ is also in $\mathscr{F}$.

Sketch of Proof. Let $d u_{g_{F}}$ denote the volume element of $g_{F}$, and let $H$ denote its Levi-Civita connection. Let $\Psi: \mathscr{M}^{*} \rightarrow R, \Psi(g)=$ $\int R(g) d \mu_{g_{F}}$. Then $\mathscr{F}_{H}^{*}$ is a critical submanifold of $\Psi$, and at the critical point $g_{F} \in \mathscr{F}_{H}^{s}$, the Hessian of $\Psi$ is given by

$$
d^{2} \Psi\left(g_{F}\right) \cdot(h, h)=-\frac{1}{2} \int(\nabla h)^{2} d_{\mu_{g_{F}}}-\frac{1}{2} \int(\nabla \operatorname{tr} h)^{2} d \mu_{g_{F}}+\int(\delta h)^{2} d \mu_{g_{F}}
$$

Let $S_{g_{F}}^{s}$ be a slice at $g_{F}($ Ebin $[3])$, and let $\Psi_{S_{t_{F}}^{*}}=\Psi \uparrow S_{g_{r}}^{s}$. Then $g_{F}$ is also a critical point for $\Psi_{S_{t_{F}}^{*}}$, and for $h \in T_{g_{F}} S_{g_{F}}^{s}=\left\{h \in S_{2}^{s}: \delta h=0\right\}$, 


$$
\begin{aligned}
d^{2} \Psi_{S_{t_{r}}}\left(g_{F}\right) \cdot(h, h)= & d^{2} \Psi\left(g_{F}\right) \cdot(h, h) \\
& =-\frac{1}{2} \int(\nabla h)^{2} d \mu_{g_{r}}-\frac{1}{2} \int(\nabla \operatorname{tr} h)^{2} d \mu_{g_{r}} .
\end{aligned}
$$

Thus $d^{2} \Psi_{S_{t_{r}}}\left(g_{F}\right)$ is negative-definite on a complement to $T_{g_{r}} \mathscr{F}_{H}^{*}=$ $\left\{h \in S_{2}^{s}: \nabla h=0\right\}$ in $T_{g_{r}} S_{g_{r}}^{s}$. Thus there exists a neighborhood $V \subset S_{g_{r}}^{s}$ of $g_{F}$ such that $\Psi_{S_{t_{r}}^{*}} \leqq 0$ on $V$, and if $\Psi_{S_{t_{p}}^{*}}(g)=0$, then $g \in \mathscr{F}_{H}$.

Let $U=\mathscr{D}^{s+1}(V)$ be the saturation of $V$. By the Slice Theorem [3], $U$ fills out a neighborhood of $g_{F}$. Thus if $g \in U$ and $R(g) \geqq 0$, there exists a $\varphi \in \mathscr{D}^{s+1}$ such that the pulled back metric $\varphi^{*} g \in$ $V \subset S_{g_{F}}^{s}$, and thus $\Psi_{s_{t_{F}}^{*}}\left(\varphi^{*} g\right) \leqq 0$. But $R\left(\varphi^{*} g\right)=R(g) \circ \varphi \geqq 0$, so that

$$
\Psi_{S_{t_{r}}^{*}}\left(\varphi^{*} g\right)=\int R\left(\varphi^{*} g\right) d \mu_{g_{r}}=\int R(g) \circ \varphi d \mu_{g_{r}} \geqq 0 .
$$

Thus $\Psi_{S_{u}^{\prime}}\left(\varphi^{*} g\right)=0$, so that $\varphi^{*} g \in \mathscr{F}_{H}^{*}$, and $g \in\left(\varphi^{-1}\right)^{*} \mathscr{F}_{H}^{*}$ is flat.

Theorem 2 is an immediate consequence of our work in $\$ 2$ and of Theorems 4 and 5.

Remarks. 1. For $\operatorname{dim} M=2$, Theorem 5 (without restricting to a neighborhood $U$ of $g_{F}$ ) is an immediate consequence of the GaussBonnet theorem and the fact that $R(g)=0 \Rightarrow g$ is flat.

2. Note that a solution $h$ of the linearized equations $D R\left(g_{F}\right) \cdot h=0$ around a flat metric $g_{F}$ is not tangent to a curve of exact solutions of $R(g)=$ 0 unless $h$ satisfies $\nabla h=0$, a condition not implied by the linearized equations. In this case where there are nonintegrable "tangent" directions, the linearized equations are said to be unstable. However, if the condition $\nabla \grave{h}=0$ is satisfied, the curve $g(t)=g_{F}+t h \in \mathscr{F}_{H}^{s},|t|$ small, $h \neq 0$, is an explicit integration of the linearized equations to a tangent curve of nonisometric flat metrics.

3. If $\mathscr{F} s=\varnothing$, then there may exist nonflat Ricci-flat metrics on $M$. The singular set $\mathscr{E}_{0}$, if not empty, has unknown structure. For example, the linearized equations are again unstable around such a metric, but we do not know if the natural extra condition on infinitesimal deformations arising from the above analysis would allow them to be integrated up to a tangent curve. This extra condition is $\int h \Delta_{L} \stackrel{\circ}{h}=0$, where $\Delta_{L}$ is the Lichnerowicz Laplacian [10].

\section{REFERENCES}

1. M. Berger and D. Ebin, Some decompositions of the space of symmetric tensors on a Riemannian manifold, J. Differential Geometry 3 (1969), 379-392. MR 42 \#993.

2. D. Brill and S. Deser, Instability of closed spaces in general relativity, Comm. Math. Phys. 32 (1973), 291-304. 
3. D. Ebin, The manifold of Riemannian metrics, Proc. Sympos. Pure Math., vol. 15, Amer. Math. Soc., Providence, R.I., 1970, pp. 11-40. MR 42 \#2506.

4. A. Fischer and J. Marsden, Linearization stability of the Einstein equations, Bull. Amer. Math. Soc. 79 (1973), 995-1001.

5. - The manifold of Riemannian metrics with prescribed scalar curvature (to appear).

6. A. Fischer and J. Wolf, The Calabi construction for compact Ricci-flat Riemannian manifolds, Bull. Amer. Math. Soc. 80 (1974), 92-97.

7. - The structure of compact Ricci-flat Riemannian manifolds (to appear).

8. J. Kazdan and F. Warner, Curvature functions for compact 2-manifolds, Ann. of Math. (to appear); See also: Bull. Amer. Math. Soc. 78 (1972), 570-574.

9. - Prescribing curvatures, AMS Summer Institute on Differential Geometry, Stanford (1973) (preprint).

10. A. Lichnerowicz, Propagateurs et commutateurs en relativité générale Inst. Hautes Études Sci. Publ. Math. No. 10 (1961), 56 pp. MR 28 \#967.

11. —, Spineurs harmoniques, C. R. Acad. Sci. Paris 257 (1963), 7-9. MR 27 \#6218.

12. J. Moser, On the volume elements on a manifold, Trans. Amer. Math. Soc. 120 (1965), 286-294. MR 32 \#409.

13. M. Obata, Certain conditions for a Riemannian manifold to be isometric with a sphere, J. Math. Soc. Japan 14 (1962), 333-340. MR 25 \#5479.

14. J. A. Wolf, Spaces of constant curvature, $2 \mathrm{~d}$ ed., J. A. Wolf, Berkeley, Calif., 1972.

Department of Mathematics, University of California, Santa Cruz, CaliFORNIA 95060

Department of Mathematics, University of California, Berkeley, California 94720 